\title{
Controlling a Submerged Rigid Body: a Geometric Analysis
}

\author{
Monique Chyba, Thomas Haberkorn, Ryan N. Smith, and George R. \\ Wilkens \\ University of Hawaii at Manoa, Mathematics Department and Ocean and \\ Resources Engineering Department, Honolulu, HI 96822 mchyba@math.hawaii.edu, \\ haberkor@math.hawaii.edu, ryan@ore.hawaii.edu, grw@math.hawaii.edu
}

Summary. In this paper we analyze the equations of motion of a submerged rigid body. Our motivation is based on recent developments done in trajectory design for this problem. Our goal is to relate some properties of singular extremals to the existence of decoupling vector fields. The ideas displayed in this paper can be viewed as a starting point to a geometric formulation of the trajectory design problem for mechanical systems with potential and external forces.

Key words: Submerged Rigid Body, Decoupling Vector Fields, Singular Trajectories

\section{Introduction}

A very important class of control systems, even though they are non-generic, is the class of controlled mechanical systems. Examples of these systems include the planar rigid body with a single variable direction thruster, the snakeboard, and underwater vehicles; see for instance [2, 9, 11]. We are interested in trajectory design problems for such systems.

All the computations in this paper are carried out on the following system: a controlled submerged rigid body. This application is particularly well adapted to our analysis for several reasons. First, there is a clear practical motivation coming from the recent trend to build autonomous underwater vehicles. The authors are currently working on implementing their techniques on a real vehicle, see [8]. The other reasons are more mathematically oriented. An underwater vehicle can be modeled as a simple mechanical control system, i.e. coming from a Lagrangian of the form kinetic minus potential energy, with dissipative forces. The uncontrolled dissipative forces reflect the damping of the system, and the restoring forces are represented in the potential forces. The corresponding system, when neglecting the external forces, has been extensively studied and thus serves as a good starting point for our analysis, 
see [3] and references therein. Clearly, the external forces introduce additional challenges for the trajectory design problem.

In $[6,7]$, we develop a numerical algorithm to compute time efficient trajectories for a controlled submerged rigid body that can be implemented on a real vehicle. The motivation and discussion of this algorithm are application oriented. We call our algorithm the Switching Time Parametrization Algorithm (STPA). These papers are application oriented. In [8] we describe experiments on a real vehicle based on this algorithm. The experiments are performed at the University of Hawaii in collaboration with the Autonomous System Laboratory from the College of Engineering. A major goal is to establish a mathematical formulation of the STPA based on the differential geometric properties of the system; such as the notion of decoupling vector fields. This paper initiates a discussion in that direction.

The key notions involved in the discussions of this paper are the ones of decoupling vector fields for invariant systems on a Lie group, namely the configuration space of a rigid body $\mathrm{SE}(3)$, and the notion of singular extremals coming from optimal control. Our analysis is based on $[1,3,4]$ for the notion of decoupling vector fields and on $[5,9]$ for the properties of singular extremals in our application.

The outline of this paper is as follows. In Section 2 we introduce the equations of motion for a controlled submerged rigid body. Section 3 introduces the definition of decoupling vector fields for an invariant system on a Lie group, and we recall the results for a controlled rigid body when neglecting the external forces. Section 4 is concerned with the application of the maximum principle to our situation and the properties of singular extremals. Our main contribution is in Section 5 where we relate the two notions introduced before.

\section{Equations of motion for underwater vehicles}

Due to subtle differences in notation and reference frames, we include a short derivation of the equations of motion for a controlled rigid body immersed in a real fluid. By real fluid we mean an ideal fluid which is not inviscid. Notice that a real fluid is assumed to be irrotational but that from a practical point of view a viscous fluid is rotational: the definition of a real fluid used in this paper is introduced for theoretical reasons. This concise derivation is based on the equations found in [10].

We will identify the position and orientation of the rigid body, with respect to the inertial frame, with an element $(b, R)$ of the Special Euclidean group of order $3 ;(b, R) \in \mathrm{SE}(3)$. We take $b=(x, y, z)^{t} \in \mathbb{R}^{3}$ to denote the position vector for the origin of the body frame, and $R \in \mathrm{SO}(3)$ as the rotation matrix describing the orientation of the body. The translational and angular velocities in the body-fixed frame are denoted by $\nu=(u, v, w)^{t}$ and $\Omega=(p, q, r)^{t}$ respectively. It follows that the kinematic equations for a rigid body are given by 


$$
\dot{b}=R \nu \text { and } \dot{R}=R \widehat{\Omega},
$$

where the operator ${ }^{\wedge}: \mathbb{R}^{3} \rightarrow \mathfrak{s o}(3)$ is defined by $\hat{y} z=y \times z ; \mathfrak{s o}(3)$ being the space of $3 \times 3$ skew-symmetric matrices. By letting $p$ be the total translational momentum, and $\pi$ the total angular momentum in the inertial frame, and $P, \Pi$ be the respective quantities in the body-fixed frame, we get that $p=R P$ and $\pi=R \Pi+\hat{b} p$. Differentiating the later two expressions, we express the dynamic equations of motion with the following:

$$
\begin{gathered}
\dot{P}=\widehat{P} \Omega+F \\
\dot{\Pi}=\widehat{\Pi} \Omega+\widehat{P} \nu+\sum_{i=1}^{k}\left(R^{t}\left(x_{i}-b\right)\right) \times R^{t} f_{i}+\tau
\end{gathered}
$$

where $F=R^{t}\left(\sum_{i=1}^{k} f_{i}\right), \tau=R^{t}\left(\sum_{i=1}^{l} \tau_{i}\right)$ represent the external forces and torques in the body-fixed frame, and $x_{i}$ is the vector from the origin of the inertial frame to the line of action of the force $f_{i}$. Next, we compute the total kinetic energy of the system in order to express equations (2) and (3) in terms of linear and angular velocities. To this end, we note that the kinetic energy of the body, $T_{b o d y}$, is given by

$$
T_{\text {body }}=\frac{1}{2}\left(\begin{array}{c}
v \\
\Omega
\end{array}\right)^{t}\left(\begin{array}{cc}
m I_{3} & -m \hat{r}_{C_{G}} \\
m \hat{r}_{C_{G}} & J_{b}
\end{array}\right)\left(\begin{array}{c}
v \\
\Omega
\end{array}\right)
$$

where $m$ is the mass of the rigid body, $I_{3}$ is the $3 \times 3$-identity matrix and $r_{C_{G}}$ is a vector that denotes the location of the body's center of gravity with respect to the origin of the body-fixed frame. The matrix $J_{b}$ represents the body moments of inertia. Based on Kirchhoff's equations we have that the kinetic energy of the fluid, $T_{\text {fluid }}$, is given by:

$$
T_{\text {fluid }}=\frac{1}{2}\left(\begin{array}{c}
v \\
\Omega
\end{array}\right)^{t}\left(\begin{array}{cc}
M_{f} & C_{f}^{t} \\
C_{f} & J_{f}
\end{array}\right)\left(\begin{array}{c}
v \\
\Omega
\end{array}\right)
$$

where $M_{f}, J_{f}$ and $C_{f}$ are respectively referred to as the added mass coefficients, the added moments of inertia coefficients and the added cross-terms. These coefficients depend on the fluid's density and on the vehicle's shape. By adding the above relations as shown, the total kinetic energy is given by:

$$
\begin{gathered}
T_{\text {body }}+T_{\text {fluid }}=T=\frac{1}{2}\left(\begin{array}{c}
v \\
\Omega
\end{array}\right)^{t} \mathbb{I}\left(\begin{array}{c}
v \\
\Omega
\end{array}\right), \\
\mathbb{I}=\left(\begin{array}{cc}
m I_{3}+M_{f} & -m \hat{r}_{C_{G}}+C_{f}^{t} \\
m \hat{r}_{C_{G}}+C_{f} & J_{b}+J_{f}
\end{array}\right)
\end{gathered}
$$

where $\mathbb{I}=\left(\begin{array}{ll}\mathbb{I}_{11} & \mathbb{I}_{12} \\ \mathbb{I}_{12}^{t} & \mathbb{I}_{22}\end{array}\right)$. Which we can simplify into:

$$
T=\frac{1}{2}\left(\nu^{t} \mathbb{I}_{11} \nu+2 \Omega^{t} \mathbb{I}_{12}^{t} \nu+\Omega^{t} \mathbb{I}_{22} \Omega\right) .
$$


Here we add the assumption that $b$, the origin of the body-fixed frame, is located at $C_{G}$; equivalently, $\hat{r}_{C_{G}}=0$. We assume the body has three planes of symmetry. Hence choosing the body axes to coincide with the principal axes of inertia implies that $J_{b}, M_{f}$ and $J_{f}$ are diagonal, while $C_{f}$ is zero. This leads to $P=\left(m I_{3}+M_{f}\right) \nu=M \nu$ and $\Pi=\left(J_{b}+J_{f}\right) \Omega=J \Omega$. It follows from equations (2) and (3) that $M \dot{\nu}=M \nu \times \Omega+F$ and $J \dot{\Omega}=$ $J \Omega \times \Omega+M \nu \times \nu+\sum_{i=1}^{k}\left(R^{t}\left(x_{i}-b\right)\right) \times R^{t} f_{i}+\tau$, where the terms $M \nu \times \Omega, J \Omega \times \Omega$ and $M \nu \times \nu$ account for the Coriolis and centripetal effects. The Coriolis and centripetal effects seen in the above equations can also be expressed in terms of $\nabla$; the Levi-Civita affine-connection for the Riemannian metric induced by the kinetic energy $T$. Explicitly, if $\gamma(t)=(b(t), R(t))$ is a curve in $\operatorname{SE}(3)$, and $\gamma^{\prime}(t)=(\nu(t), \Omega(t))$ is its pseudo-velocity (1), then

$$
\nabla_{\gamma^{\prime}} \gamma^{\prime}=\left(\begin{array}{c}
\dot{\nu}+M^{-1}(\Omega \times M \nu) \\
\dot{\Omega}+J^{-1}(\Omega \times J \Omega+\nu \times M \nu)
\end{array}\right) .
$$

Let us now discuss the external forces acting on a submerged rigid body. We assume that the vehicle is neutrally buoyant, which means that the buoyancy force and the gravitational force cancel each other. Since the origin of the body-fixed frame is $C_{G}$, the only restoring force acting on the vehicle is the torque from the buoyancy force induced upon listing, $r_{C_{B}} \times R^{t} \rho g \nu k$. Here $r_{C_{B}}$ is the vector from $C_{G}$ to $C_{B}$, where $\rho$ is the fluid density, $g$ the acceleration of gravity, $v$ the volume of fluid displaced by the rigid body and $k$ the unit vector pointing in the direction of gravity. Additional hydrodynamic forces experienced by the rigid body are due to drag effects. We make the assumption that we have a drag force $D_{\nu}(\nu)$ and a drag momentum $D_{\Omega}(\Omega)$ quadratic in the velocities and we neglect the off-diagonal terms. We summarize our computations in the following definition.

Definition 1. Under our assumptions, the equations of motion, in the bodyfixed frame, for a rigid body submerged in a real fluid are given by:

$$
\begin{aligned}
M \dot{\nu}= & M \nu \times \Omega+D_{\nu}(\nu) \nu+F \\
J \dot{\Omega}= & J \Omega \times \Omega+M \nu \times \nu \\
& +D_{\Omega}(\Omega) \Omega-r_{C_{B}} \times R^{t} \rho g \nu k+\tau
\end{aligned}
$$

where $M$ accounts for the mass of the rigid body and the added mass coefficients, $J$ accounts for the body moments of inertia and the added moments of inertia coefficients. The matrices $D_{\nu}(\nu), D_{\Omega}(\Omega)$ represent respectively the drag force and momentum, while the restoring force acting on the body is due to the torque induced by the buoyancy force. Finally, $F=\left(f_{1}, f_{2}, f_{3}\right)^{t}$ and $\tau=\left(\tau_{1}, \tau_{2}, \tau_{3}\right)^{t}$ account for the control.

Section 3 of this paper deals with the geometric properties of the system. In that section the domain of control is assumed to be unbounded, thus allowing us to identify some important geometric structures. Once these structures 
are identified, we will discard the unbounded assumption since the controls represent forces with limited power, such as thrusters. Subsequent sections will, for simplicity, assume the domain of control to be $\mathcal{F}=\left\{f \in \mathbb{R}^{3} \mid-1 \leq\right.$ $\left.f_{1,2,3} \leq 1\right\}, \mathcal{T}=\left\{\tau \in \mathbb{R}^{3} \mid-1 \leq \tau_{1,2,3} \leq 1\right\}$. An admissible control is a measurable bounded function $\varphi:[0, T] \rightarrow \mathcal{U}=\mathcal{F} \times \mathcal{T}$.

A final remark concerns the equations of motion of a rigid body moving in the air. In this case, the dissipation term due to the drag and the restoring forces are negligible. Moreover, since the density of air is much smaller than that of water we can neglect the terms due to the added mass tensor. We are then left with the well known equations of motion of a rigid body moving in the air. These equations can serve as a first approximation when considering a real fluid.

\section{Affine-Connection control systems and decoupling vector fields}

Together, equations (1) and (10) form a first-order affine control system on the tangent bundle $T \mathrm{SE}(3)$ that represents the second-order forced affineconnection control system on $\mathrm{SE}(3)$

$$
\nabla_{\gamma^{\prime}} \gamma^{\prime}=\left(\begin{array}{c}
M^{-1}\left(D_{\nu}(\nu) \nu+F\right) \\
J^{-1}\left(D_{\Omega}(\Omega) \Omega-r_{C_{B}} \times R^{t} \rho g \nu k+\tau\right)
\end{array}\right) .
$$

In the absence of a restoring force $r_{C_{B}} \times R^{t} \rho g \nabla k$, a drag momentum $D_{\Omega}(\Omega) \Omega$, and a drag force $D_{\nu}(\nu) \nu$ the equations of motion (11) represent a left-invariant affine-connection control system on the Lie group $\mathrm{SE}(3)$,

$$
\nabla_{\gamma^{\prime}} \gamma^{\prime}=\left(\begin{array}{c}
M^{-1} F \\
J^{-1} \tau
\end{array}\right)
$$

Definition 2. An affine-connection control system on a manifold $Q$ is determined by an affine-connection, $\nabla$, and a constant-rank distribution $y \subset T Q . A$ trajectory for the system is a curve $\gamma:[0, T] \rightarrow Q$ such that $\gamma^{\prime}:[0, T] \rightarrow T Q$ is absolutely continuous, $\gamma^{\prime}(0)=0 \in T_{\gamma(0)} Q$, and $\nabla_{\gamma^{\prime}(t)} \gamma^{\prime}(t) \in y_{\gamma(t)}$ for almost all $t \in[0, T]$.

A common presentation for such a system is $\nabla_{\gamma^{\prime}(t)} \gamma^{\prime}(t)=\sum_{a=1}^{k} u^{a}(t) Y_{a}(\gamma(t))$, where $u^{1}(t), \ldots, u^{k}(t)$ are measurable controls and $Y_{1}, \ldots, Y_{k}$ are independent vector-fields on $Q$ that span $y$.

Just as equation (11) on $\mathrm{SE}(3)$ is equivalent to equations (1) and (10) on $T \mathrm{SE}(3)$, an affine-connection control system on $Q$ is equivalent to an affine control system on $T Q$. The equivalence is realized via the geodesic spray of an affine-connection and the vertical lift of tangent vectors to $Q$. 
Definition 3. Let $v \in T_{q} Q \subset T Q$, then the vertical lift at $v$ is a map $\operatorname{vlft}_{v}: T_{q} Q \rightarrow T_{v} T Q$. For $w \in T_{q} Q$, we define $\operatorname{vlft}_{v}(w)=\left.\frac{d}{d t}(v+t w)\right|_{t=0}$. In components, $\operatorname{vlft}_{v}(w)=\left(\begin{array}{c}0 \\ w\end{array}\right) \in T_{v} T Q$.

Definition 4. The geodesic spray of $\nabla$ is the vector field $S$, on $T Q$, that generates geodesic flow. Specifically, for $v \in T_{q} Q, S(v)=\left.\frac{d}{d t} \gamma_{v}^{\prime}(t)\right|_{t=0}$ where $\gamma_{v}$ is the unique $\nabla$-geodesic such that $\gamma_{v}(0)=q$ and $\gamma_{v}^{\prime}(0)=v$.

In the special case of our Levi-Civita connection (9),

$$
S(b, R, \nu, \Omega)=\left(\begin{array}{c}
\nu \\
\Omega \\
-M^{-1}(\Omega \times M \nu) \\
-J^{-1}(\Omega \times J \Omega+\nu \times M \nu)
\end{array}\right) .
$$

For this presentation of $S(b, R, \nu, \Omega)$, the components are expressed relative to the standard left-invariant basis of vector fields on $T \mathrm{SE}(3)$ rather than coordinate vector fields. Equation (1) can be used to recover expressions for $\dot{b}$ and $\dot{R}$.

Given an affine-connection control system $\nabla_{\gamma^{\prime}} \gamma^{\prime}=\sum_{a=1}^{k} u^{a}(t) Y_{a}(\gamma(t))$ on $Q$, we associate to it the following affine control system: $\Upsilon^{\prime}(t)=S(\Upsilon(t))+$ $\sum_{a=1}^{k} u^{a}(t) \operatorname{vlft}\left(Y_{a}\right)$ on $T Q$. In [3, p224] the authors show that trajectories for the affine-connection control system on $Q$ map bijectively to trajectories for the affine control system on $T Q$ whose initial points lie on the zero-section. The bijection maps the trajectory $\gamma:[0, T] \rightarrow Q$ to the trajectory $\Upsilon=\gamma^{\prime}$ : $[0, T] \rightarrow T Q$.

Definition 5. $A$ decoupling vector field for an affine-connection control system is a vector field $V$ on $Q$ having the property that every reparametrized integral curve for $V$ is a trajectory for the affine-connection control system. More precisely, let $\gamma:[0, S] \rightarrow Q$ be a solution for $\gamma^{\prime}(s)=V(\gamma(s))$ and let $s:[0, T] \rightarrow[0, S]$ satisfy $s(0)=s^{\prime}(0)=s^{\prime}(T)=0, s(T)=S, s^{\prime}(t)>0$ for $t \in(0, T)$, and $(\gamma \circ s)^{\prime}:[0, T] \rightarrow T Q$ is absolutely continuous. Then $\gamma \circ s:[0, T] \rightarrow Q$ is a trajectory for the affine-connection control system.

A necessary and sufficient condition for $V$ to be a decoupling vector field is that both $V$ and $\nabla_{V} V$ are sections of $y$ [3, p. 426]. Notice that if $y=T Q$ then every vector field is a decoupling vector field, and if $y=\operatorname{Span}\{Y\}$ then $V$ is a decoupling vector field if and only if both $V$ and $\nabla_{V} V$ are multiples of $Y$.

Decoupling vector fields for an under-actuated system (12) are analyzed in [4]. In the under-actuated setting, decoupling vector fields are found by solving a system of homogeneous quadratic polynomials in several variables. For our model, the control forces $F=\left(f_{1}, f_{2}, f_{3}\right)^{t}$ and $\tau=\left(\tau_{1}, \tau_{2}, \tau_{3}\right)^{t}$ are unconstrained; the model is a fully-actuated affine-connection control system. In this case there are no quadratic polynomials to solve and every left-invariant vector 
field is a decoupling vector field. Something interesting can occur, however, when we seek single-input subsystems that admit a decoupling vector field. Let us start with a single-input affine-connection control system, where the input vector field is $Y=(F, \tau)^{t}$. As mentioned above, to be decoupling for $y=\operatorname{Span}\{Y\}$ a vector field $V$, as well as $\nabla_{V} V$, must be a multiple of $Y$.

Proposition 1. Let $\nabla$ be the affine connection (9) and consider the single input affine-connection control system $\nabla_{\gamma^{\prime}} \gamma^{\prime}=u(t) Y(\gamma(t))$, where $Y=(F, \tau)^{t}$. Then $V=(F, \tau)^{t}$ is a decoupling vector field for $Y$ if and only if $V$ is a multiple of $\left(e_{i}, e_{i}\right)^{t}$, where $e_{1}, e_{2}, e_{3}$ is the standard basis for $\mathbb{R}^{3}$, or all but one of its components are zero.

Proof. The conditions for $V$ to be decoupling eventually reduce to finding an eigenvector belonging to a real eigenvalue for the following matrix:

$$
\left(\begin{array}{cc}
-\hat{\tau} & 0 \\
-\widehat{F} & -\hat{\tau}
\end{array}\right)\left(\begin{array}{cc}
M & 0 \\
0 & J
\end{array}\right)\left(\begin{array}{l}
F \\
\tau
\end{array}\right)=\lambda\left(\begin{array}{cc}
M & 0 \\
0 & J
\end{array}\right)\left(\begin{array}{l}
F \\
\tau
\end{array}\right)
$$

It is equivalent to $-\hat{\tau} M F=\lambda M F$ and $-\hat{F} M F-\hat{\tau} J \tau=\lambda J \tau$. Since $\lambda$ is real and $\hat{\tau}$ is skew-symmetric, $\lambda=0$. If $F$ is zero, we have that $\hat{\tau} J \tau=\tau \times J \tau=0$. We see that $V=(0, \tau)^{t}$ will be a decoupling vector field for $Y=(0, \tau)^{t}$ provided $\tau$ is an eigenvector for $J$. Similarly, when $\tau=0$, then we must have $\lambda=0$ and $\widehat{F} M F=F \times M F=0$. We see that $V=(F, 0)^{t}$ will be a decoupling vector field for $Y=(F, 0)^{t}$ provided $F$ is an eigenvector for $M$. Since $M$ and $J$ are diagonal matrices, the eigenvectors are precisely those for which all but one of $\left\{f_{1}, f_{2}, f_{3}, \tau_{1}, \tau_{2}, \tau_{3}\right\}$ are zero. Assume now $F \neq 0$. It follows that $M F=\mu \tau$ where $\mu$ is some constant and $-\hat{F} M F-\hat{\tau} J \tau=0$. This last equality is equivalent to $\tau \times(J \tau-\mu F)=0$ or in other words $J \tau-\mu F=\alpha \tau$ where $\alpha$ is a constant. By using the two previous relations between $F$ and $\tau$, we have that $\left(J-\mu^{2} M^{-1}\right) \tau=\alpha \tau$. Since $J-\mu^{2} M^{-1}$ is diagonal, $\tau$ is an eigenvector if all but one of its component are zero. The same is deduced them for $F$.

We note that if we consider the case when all but one of the $\left\{f_{1}, f_{2}, f_{3}, \tau_{1}, \tau_{2}, \tau_{3}\right\}$ are zero, these decoupling vector fields are exactly the pure motions for a rigid body moving in air, and were investigated in [7].

\section{Singular extremals}

Our goal is to establish a relation between singular extremals arising in optimal control and the conditions for a vector field to be decoupling. To this end, we first recall the notion of singular extremals.

Consider the minimum time problem for a controlled submerged rigid body. We assume the domain of control to be as stated in Section 2. Since the necessary conditions of the maximum principle are local, we assume the equations of motion expressed in local coordinates found in [7]. 
We introduce $\psi$, a triple of Euler angles for $R \in \mathrm{SO}(3) ; \eta=(b, \psi)$, local coordinates for $(b, R) \in \mathrm{SE}(3) ; \chi=(\eta, \nu, \Omega)$, local coordinates for $T \mathrm{SE}(3)$; and let $\chi_{0}=\chi(0)$ and $\chi_{T}=\chi(T)$ be the initial and final states for our submerged rigid body. We let $\Theta$ be the $3 \times 3$ matrix for which $\dot{\psi}=\Theta \Omega$ and, as always, $\dot{b}=R \nu$. Note first, that the equations of motion derived in the previous section can be written as an affine control system:

$$
\dot{\chi}(t)=Y_{0}(\chi(t))+\sum_{i=1}^{6} Y_{i}(t) \varphi_{i}(t)
$$

where $\varphi=(F, \tau)^{t}$ is the control and the drift $Y_{0}$ is given by

$$
Y_{0}=\left(\begin{array}{c}
R \nu \\
\Theta \Omega \\
M^{-1}\left[M \nu \times \Omega+D_{\nu}(\nu) \nu\right] \\
J^{-1} a_{41}
\end{array}\right)
$$

with $a_{41}=J \Omega \times \Omega+M \nu \times \nu+D_{\Omega}(\Omega) \Omega-r_{C_{B}} \times R^{t} \rho g$. Notice that when neglecting the external forces, the drift is the geodesic spray. The input vector fields are given by:

$$
Y_{i}=\operatorname{vlft}\left(\mathbb{I}_{i}^{-1}\right)=\left(\begin{array}{c}
0 \\
0 \\
\mathbb{I}_{i}^{-1}
\end{array}\right)
$$

with $\mathbb{I}_{i}^{-1}$ being the $i^{\text {th }}$ column of the matrix $\mathbb{I}^{-1}=\left(\begin{array}{cc}M^{-1} & 0 \\ 0 & J^{-1}\end{array}\right)$.

Assume that there exists an admissible time-optimal control $\varphi:[0, T] \rightarrow$ $\mathcal{U}$, such that the corresponding trajectory $\chi=(\eta, \nu, \Omega)$ is a solution of the equations of motion, in Section 2, and steers the body from $\chi_{0}$ to $\chi_{T}$. The Maximum Principle, see [12], implies that there exists an absolutely continuous vector $\lambda=\left(\lambda_{\eta}, \lambda_{\nu}, \lambda_{\Omega}\right):[0, T] \rightarrow \mathbb{R}^{12}, \lambda(t) \neq 0$ for all $t$, such that the following conditions hold almost everywhere:

$$
\begin{array}{r}
\dot{\eta}=\frac{\partial H}{\partial \lambda_{\eta}}, \dot{\nu}=\frac{\partial H}{\partial \lambda_{\nu}}, \dot{\Omega}=\frac{\partial H}{\partial \lambda_{\Omega}}, \\
\dot{\lambda}_{\eta}=-\frac{\partial H}{\partial \eta}, \dot{\lambda}_{\nu}=-\frac{\partial H}{\partial \nu}, \dot{\lambda}_{\Omega}=-\frac{\partial H}{\partial \Omega}
\end{array}
$$

where the Hamiltonian function $H$ is given by

$$
\begin{aligned}
H(\chi, \lambda, \varphi) & =\lambda_{\eta}^{t}(R \nu, \Theta \Omega)^{t}+\lambda_{\nu}^{t} M^{-1}\left[M \nu \times \Omega+D_{\nu}(\nu) \nu+F\right] \\
& +\lambda_{\Omega}^{t} J^{-1}\left[J \Omega \times \Omega+M \nu \times \nu+D_{\Omega}(\Omega) \Omega-r_{C_{B}} \times R^{t} \rho g \nabla k+\tau\right] .
\end{aligned}
$$

Additionally, the maximum condition

$$
H(\chi(t), \lambda(t), \varphi(t))=\max _{\gamma \in \mathcal{U}} H(\chi(t), \lambda(t), \gamma)
$$


holds for almost every $t$. The maximum of the Hamiltonian is constant along the solutions of (17), (18) and must satisfy $H(\chi(t), \lambda(t), \varphi(t))=\lambda_{0}, \lambda_{0} \geq 0$. A triple $(\chi, \lambda, \varphi)$ that satisfies the Maximum Principle is called an extremal, and the vector function $\lambda$ is called the adjoint vector.

The component $\phi_{i}, i=1, \ldots, 6$, of the vectors $\lambda_{\nu}^{t} M^{-1}$ and $\lambda_{\Omega}^{t} J^{-1}$ are called the switching functions. The maximum condition, along with the control domain $\mathcal{U}$ and the form of the equations of motion, is equivalent almost everywhere to $\varphi_{i}(t)=-1$ if $\phi_{i}(t)<0$ and $\varphi_{i}(t)=+1$ if $\phi_{i}(t)>0$ for $i=1, \ldots, 6$. Notice that from our assumptions on the fluid and the body, the matrices $M$ and $J$ are diagonal. It follows that the switching functions are, modulo a constant, the last six components of the adjoint vector, and are absolutely continuous. Clearly, the zeros of the switching functions determine the structure of the extremals. We are interested in the case when a switching function is identically zero on a non trivial interval $\left[t_{1}, t_{2}\right]$. We say that the extremal is singular in the corresponding control on this interval. To compute a singular control $\varphi_{i}$ we must differentiate the switching function $\phi_{i}$.

We finish the section with these computations. By construction, we have that $\phi_{i}(t)=\lambda^{t}(t) Y_{i}$. It is then a standard fact that the derivative $\dot{\phi}_{i}$ along an extremal is given by:

$$
\dot{\phi}_{i}(t)=\lambda^{t}(t)\left[Y_{0}, Y_{i}\right](\chi(t))+\sum_{j=1}^{6} \lambda^{t}(t)\left[Y_{j}, Y_{i}\right](t) \varphi_{j}(t)
$$

where [ , ] denotes the Lie bracket of vector fields. Since the vector fields $Y_{i}$ are constants, their Lie brackets are zero and the switching functions have absolutely continuous derivatives: $\dot{\phi}(t)=\lambda^{t}(t)\left[Y_{0}, Y_{i}\right](\chi(t))$. Differentiating once more, we obtain

$$
\ddot{\phi}_{i}(t)=\lambda^{t}(t) \operatorname{ad}_{Y_{0}}^{2} Y_{i}(\chi(t))+\sum_{j=1}^{6} \lambda^{t}(t)\left[Y_{j},\left[Y_{0}, Y_{i}\right]\right](\chi(t)) \varphi_{j}(t)
$$

Proposition 2. For a rigid body moving in the air, the following holds:

$$
\left[Y_{i},\left[Y_{0}, Y_{i}\right]\right](\chi) \equiv 0, \quad i=1, \ldots, 6 .
$$

In a real fluid, the previous Lie bracket is not zero but satisfies:

$$
\left[Y_{i},\left[Y_{0}, Y_{i}\right]\right](\chi) \in \operatorname{Span}\left\{Y_{i}\right\}, \quad i=1, \ldots, 6 .
$$

Proof. A first remark is that only quadratic terms with respect to the velocities in $Y_{0}$ can produce a non zero component for $\left[Y_{j},\left[Y_{0}, Y_{i}\right]\right](\chi(t))$. Hence we have that $\left[Y_{j},\left[Y_{0}, Y_{i}\right]\right](\chi) \in \operatorname{Span}\left\{Y_{1}, \ldots, Y_{6}\right\}$. Moreover, it is an easy verification that under our assumptions neither $M \nu \times \Omega, J \Omega \times \Omega$ nor $M \nu \times \nu$ contains terms of the form $u^{2}, v^{2}, w^{2}, p^{2}, q^{2}, r^{2}$. We can conclude that for a rigid body moving in air (or more generally when we neglect the external forces) the Lie 
brackets $\left[Y_{i},\left[Y_{0}, Y_{i}\right]\right](\chi)$ are identically zero for $i=1, \ldots, 6$. In a real fluid, this deduction is not possible since the drag terms, as assumed in this paper, will produce a non zero component. However, it can be verified that in a real fluid the only non zero component of $\left[Y_{i},\left[Y_{0}, Y_{i}\right]\right](\chi)$ is a multiple of $Y_{i}$. Note that the restoring forces play no role in the value of this Lie bracket.

Equations (20) and (21) can be interpreted in terms of the order of singular extremals. Along a $\varphi_{i}$-singular arc, let $q$ be such that $\frac{d^{2 q}}{d t^{2 q}} \phi_{i}$ is the lowest order derivative in which $\varphi_{i}$ appears explicitly with a nonzero coefficient. We define $q$ as the order of the singular control $\varphi_{i}$. This definition assumes the well known result that a singular control $\varphi_{i}$ first appears explicitly in an even order derivative of $\phi_{i}$, see [13].

Proposition 3. Let $\chi$ be an extremal that is singular for the component $\varphi_{i}$ of the control while the other ones are bang. Then, for a submerged rigid body moving in air or in a real fluid, the singular control is of order at least 2.

Proof. Let us assume $\chi$ is an extremal that is singular for the component $\varphi_{i}$ of the control while the other ones are constant, i.e. the function $\phi_{i}$ is identically zero along the extremal. We can compute the singular control from equation (20) as long as the term $\lambda^{t}\left[Y_{i},\left[Y_{0}, Y_{i}\right]\right](\chi)$ is non zero. From Proposition 2, this Lie bracket is zero for a rigid body moving in air and a multiple of $Y_{i}$ for a real fluid. Since along the extremal $\phi_{i}=\lambda^{t} Y_{i}=0$ the result is true in a real fluid as well. This means that we must compute at least the fourth derivative of the switching function to obtain the singular control as a feedback.

\section{Main observation}

The main result consists of an observation that suggests directions for further study. The observation concerns a possible relationship between singular extremals of order greater than 1 and decoupling vector fields. As seen above, for a rigid body moving in the air the order of the singular control $\varphi_{i}$ is greater than 1 since it satisfies the condition $\left[Y_{i},\left[Y_{0}, Y_{i}\right]\right](\chi) \equiv 0$. Note that this also implies that $Y_{i}$ is a decoupling vector field for the single-input affine control system where every control is set to 0 except for $\varphi_{i}$. This last result was already proved in Proposition 1 using an ad hoc method without referring to singular extremals or differential geometric properties of the system. To see the relation between equation 20 and Proposition 1 we note that $Y_{i}$ is the vertical lift of the control $(F, \tau)=(0, \ldots, 1, \ldots, 0)$, where the 1 is in the $i$ th position. We also note that for a rigid body moving in the air $Y_{0}(14)$ is the geodesic spray for the Levi-Civita affine connection (9). The final ingredient is the easily verifiable equation $[\operatorname{vlft}(X),[S, \operatorname{vlft}(X)]]=\operatorname{vlft}\left(2 \nabla_{X} X\right)$. Hence $\left[Y_{i},\left[Y_{0}, Y_{i}\right]\right](\chi) \equiv 0$ says that $\nabla_{Y_{i}} Y_{i}=0$, where we abuse notation by using $Y_{i}$ to also denote the vector field whose vertical lift is $Y_{i}$. So $Y_{i}$ being autoparallel implies that it is decoupling and that $\varphi_{i}$ has order greater than one. 
This establishes a relationship between the condition that $V$ is a decoupling vector field if both $V$ and $\nabla_{V} V$ are sections of $y$ and Lie bracket properties of singular extremals for the time minimal problem.

For a forced affine-connection control system, such as a submerged rigid body in a real fluid, we should be somewhat cautious since the notion of a decoupling vector field is not clearly defined. Singular extremals, however, certainly are and the condition $\left[Y_{i},\left[Y_{0}, Y_{i}\right]\right](\chi) \in \operatorname{Span}\left\{Y_{i}\right\}$ still holds in our case. This perhaps suggests a proper generalization and characterization of the notion of a decoupling vector field for forced affine-connection control systems.

\section{Thanks}

The authors would like to warmly thank A.D. Lewis for very illuminating discussions and suggestions.

\section{References}

1. F. Bullo. Trajectory design for mechanical systems: from geometry to algorithms. European Journal of Control, 10(5):397-410, 2004.

2. F. Bullo, N.E. Leonard, and A.D. Lewis. Controllability and motion algorithms for underactuated lagrangian systems on lie groups. Institute of Electrical and Electronics Engineers. Transactions on Automatic Control, 45(8):1437-1454, 2000 .

3. F. Bullo and A.D. Lewis. Geometric Control of Mechanical Systems. Number 49 in Texts in Applied Mathematics, New York-Heidelberg-Berlin, 2004.

4. F. Bullo and K.M. Lynch. Kinematic controllability for decoupled trajectory planning in underactuated mechanical systems. IEEE Trans. Robotics and Automation, 17(4):402-412, 2001.

5. M. Chyba and T. Haberkorn. Designing efficient trajectories for underwater vehicles using geometric control theory. Proceedings of the 24th International Conference on Offshore Mechanics and Artic Enginnering, 2005.

6. M. Chyba and T. Haberkorn. Optimization techniques for autonomous underwater vehicles: a practical point of view. Proceedings of the 17th International Symposium on Mathematical Theory of Networks and Systems, 2006.

7. M. Chyba, T. Haberkorn, R.N. Smith, and S.K. Choi. Implementable efficient time and energy consumption trajectories design for an autonomous underwater vehicle. $6^{\text {th }}$ Conf. Computer and IT Applications in the Maritime Industries (COMPIT), Cortona, pages 443-457, 2007.

8. M. Chyba, T. Haberkorn, R.N. Smith, S. Zhao, and S.K. Choi. Towards practical implementation of time optimal trajectories for underwater vehicles. Proceedings of the 25th International Conference on Offshore Mechanics and Artic Enginnering, 2006.

9. M. Chyba, N.E. Leonard, and E.D. Sontag. Singular trajectories in multi-input time-optimal problems: Application to controlled mechanical systems. J. of Dynamical and Control Systems, 9:73-88, 2003. 
10. N. E. Leonard. Stability of a bottom-heavy underwater vehicle. Automatica, 33:331-346, 1997.

11. A. D. Lewis, J.P. Ostrowski, R.M. Murray, and J.W. Burdick. Nonholonomic mechanics and locomotion: the snakeboard example. Proceedings of the IEEE International Conference on Robotics and Automation, Institute of Electrical and Electronics Engineers, pages 2391-2400, 1994.

12. L. S. Pontryagin et al. The mathematical theory of optimal processes, volume 55 of International series of monographs in pure and applied mathematics. Macmillan, 1964. Translated by D.E. Brown.

13. H.M. Robbins. A generalized Legendre-Clebsch condition for the singular cases of optimal control. IBM Journal of Research and Development, 11(4):361-372, 1967. 